\title{
AN OPTIMIZATION OF THE BESICOVITCH COVERING
}

\author{
PETER A. LOEB
}

(Communicated by Andreas R. Blass)

\begin{abstract}
Given an appropriate covering by balls of a set in a metric space, we construct an optimized version of the subcovering used in the proof of Besicovitch's theorem. The proof is nonstandard and suggests a general method for optimizing standard geometric constructions.
\end{abstract}

In this note, we construct an optimized version of the covering by balls used in the proof of the Besicovitch covering theorem ([1]; also see [3]). That is, given an arbitrary set $A$ in an appropriate metric space and a covering $\{B(a, r(a))$ : $a \in A\}$ by closed balls with bounded radii, we want to extract a subcovering with no center in the interior of any other ball of the subcovering and each point $a \in A$ in a ball with radius no smaller than $r(a)$. Easily constructed examples on the real line show that in the absence of additional assumptions on the radius function $r(\cdot)$, we must adjoin limit balls to the original covering. The proof that the desired subcovering then exists is nonstandard. David Berg has shown that a standard diagonalization argument works for $\sigma$-compact spaces. The proof given here uses the standard part map after replacing a magnification factor in the standard Besicovitch covering with a factor infinitely close to 1 . This suggests a general method for optimizing standard geometric constructions.

Our setting is a metric space $(X, \rho)$; we write $B(c, r)$ to denote a closed metric ball $\{x \in X: \rho(c, x) \leq r\}$ and $\mathrm{Cl}(E)$ to denote the closure of a set $E$ in $X$. We will assume a local compactness condition that is trivially satisfied if closed balls in $X$ are compact.

Theorem. Let $A$ be an arbitrary subset of $X$. Assume that at each point $a \in A$ there is centered a closed ball $B(a, r(a))$ with positive radius so that $\sup _{a \in A} r(a)$ $<+\infty$. Also assume that for each $c \in A$, the set

$$
K(c)=\mathrm{Cl}(\{a \in A: c \in B(a, r(a))\})
$$

is compact. With each point $p \in \mathrm{Cl}(A)$ we associate the set

$$
L(p)=\{t>0: \forall \varepsilon>0, \exists a \in A \text { with } \rho(p, a)<\varepsilon \text { and }|t-r(a)|<\varepsilon\} .
$$

There exists a collection of closed balls $\mathscr{B}$ with

$$
\mathscr{B} \subseteq\{B(p, t): p \in \mathrm{Cl}(A) \text { and } t \in L(p)\}
$$

Received by the editors September 23, 1991 and, in revised form, November 4, 1991.

1991 Mathematics Subject Classification. Primary 03H05, 28A75, 52C17.

Key words and phrases. Besicovitch, optimized covering, nonstandard analysis.

This work was supported in part by a grant from the U. S. National Science Foundation.

(c) 1993 American Mathematical Society $0002-9939 / 93 \$ 1.00+\$ .25$ per page 
such that no center of any ball in $\mathscr{B}$ is in the interior of any other ball in $\mathscr{B}$, and each $a \in A$ is in a ball $B(p, t) \in \mathscr{B}$ with $t \geq r(a)$. If the radius function $r$ is continuous on $A$ and $\lim _{a \rightarrow p} r(a)=0$ at every point $p \in \operatorname{Cl}(A) \backslash A$, then $\mathscr{B} \subseteq\{B(a, r(a)): a \in A\}$.

Proof. Given a real number $s>1$, one may choose a point $a_{1} \in A$ so that for each $a \in A, s \cdot r\left(a_{1}\right)>r(a)$. Setting $A_{1}=A \backslash B\left(a_{1}, r\left(a_{1}\right)\right)$, there is an $a_{2} \in A_{1}$ so that for all $a \in A_{1}, s \cdot r\left(a_{2}\right)>r(a)$. By induction over the ordinals (see, for example, [3]), one may continue in this way to obtain a well-ordered subset $\left\{a_{\alpha}\right\}$ of $A$ such that $A \subseteq \bigcup_{\alpha} B\left(a_{\alpha}, r\left(a_{\alpha}\right)\right)$. This is a Besicovitch covering of $A$. We will employ the transfer principle to obtain a set with the properties of $\left\{a_{\alpha}\right\}$ in a nonstandard structure containing $X$ (see [2]). Fix $s=1+\varepsilon$ where $\varepsilon$ is a positive infinitesimal. There is an internal subset $A_{0}=\left\{a_{\alpha}\right\}$ of ${ }^{*} A$ with an internal well-ordering $\preceq$ such that for $r_{\alpha}={ }^{*} r\left(a_{\alpha}\right)$ we have ${ }^{*} A \subseteq \bigcup_{\alpha} B\left(a_{\alpha}, r_{\alpha}\right)$, and when $a_{\alpha} \supsetneqq a_{\beta}, a_{\beta} \notin B\left(a_{\alpha}, r_{\alpha}\right)$ and $s \cdot r_{\alpha}>r_{\beta}$. Moreover, if $a$ is in ${ }^{*} A$ and $a_{\alpha}$ is the first element of $A_{0}$ with $a \in B\left(a_{\alpha}, r_{\alpha}\right)$, then $s \cdot r_{\alpha}>{ }^{*} r(a)$. Suppose $a_{\alpha} \in A_{0}$ and $B\left(a_{\alpha}, r_{\alpha}\right)$ contains a standard point $c \in A$. Then $a_{\alpha}$ is in the nonstandard extension of the compact set $K(c)$, so $a_{\alpha}$ is infinitely close in terms of the internal metric ${ }^{*} \rho$ to a standard point ${ }^{\circ} a_{\alpha} \in K(c) \subseteq \mathrm{Cl}(A)$. If the standard part ${ }^{\circ} r_{\alpha}$ of $r_{\alpha}$ is strictly positive, then ${ }^{\circ} r_{\alpha}$ is in $L\left({ }^{\circ} a_{\alpha}\right)$. Set

$$
\mathscr{B}=\left\{B\left({ }^{\circ} a_{\alpha},{ }^{\circ} r_{\alpha}\right): a_{\alpha} \in A_{0},{ }^{\circ} r_{\alpha}>0 \text { and } \exists c \in A \text { with } c \in B\left(a_{\alpha}, r_{\alpha}\right)\right\} \text {. }
$$

To see this is a covering of $A$ with the desired properties, fix balls $B\left({ }^{\circ} a_{\alpha},{ }^{\circ} r_{\alpha}\right)$ and $B\left({ }^{\circ} a_{\beta},{ }^{\circ} r_{\beta}\right)$ in $\mathscr{B}$ with $a_{\alpha} \supsetneqq a_{\beta}$. Then $a_{\beta} \notin B\left(a_{\alpha}, r_{\alpha}\right)$ and $(1+\varepsilon) \cdot r_{\alpha} \geq$ $r_{\beta}$, so

$$
\rho\left({ }^{\circ} a_{\beta},{ }^{\circ} a_{\alpha}\right) \geq{ }^{\circ} r_{\alpha} \geq{ }^{\circ} r_{\beta}>0 \text {. }
$$

If $c \in A$ and if $a_{\alpha}$ is the first element of $A_{0}$ with $c \in B\left(a_{\alpha}, r_{\alpha}\right)$, then $\rho\left(c,{ }^{\circ} a_{\alpha}\right)$ $\leq{ }^{\circ} r_{\alpha}$, so $c \in B\left({ }^{\circ} a_{\alpha},{ }^{\circ} r_{\alpha}\right)$, and $(1+\varepsilon) \cdot r_{\alpha}>r(c)$, so ${ }^{\circ} r_{\alpha} \geq r(c)>0$.

There may be some redundancy in the collection $\mathscr{B}$. We can, of course, remove a ball $B\left({ }^{\circ} a_{\beta},{ }^{\circ} r_{\beta}\right)$ when $B\left({ }^{\circ} a_{\beta},{ }^{\circ} r_{\beta}\right) \subseteq \bigcup\left\{B\left({ }^{\circ} a_{\alpha},{ }^{\circ} r_{\alpha}\right) \in \mathscr{B}: a_{\alpha} \supsetneqq a_{\beta}\right\}$. The proof given here illustrates the use of the transfer principle in optimizing a standard construction. Hermann Render has noted that the proof can also begin by fixing a hyperfinite set $A_{f}$ containing every standard point in $A$, and successively selecting a ball with maximum radius and center in $A_{f}$ from balls with centers not yet covered in $A_{f}$.

\section{REFERENCES}

1. A. S. Besicovitch, A general form of the covering principle and relative differentiation of additive functions I, Proc. Cambridge Philos. Soc. 41 (1945), 103-110; II, 42 (1946), 1-10.

2. A. E. Hurd and P. A. Loeb, An introduction to nonstandard real analysis, Pure Appl. Math., vol. 118, Academic Press, Orlando, FL, 1985.

3. P. A. Loeb, On the Besicovitch covering theorem, Tokyo J. Math 25 (1989), 51-55.

\footnotetext{
Department of Mathematics, University of Illinois, 1409 West Green Street, Urbana, ILLINOIS 61801

E-mail address: loeb@uiucmath.math.uiuc.edu
} 